\title{
P-0559 Prevalence of low serum testosterone levels among men with type 2 diabetes mellitus attending two outpatient diabetes clinics in Durban, South Africa
}

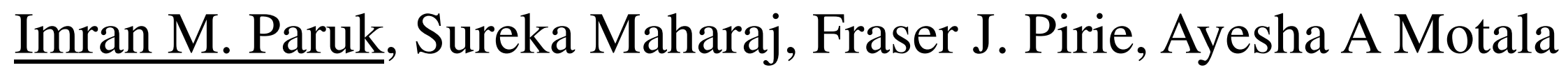

\section{Department of Diabetes and Endocrinology, Inkosi Albert Luthuli Hospital (IALCH), Nelson R Mandela School of Medicine, University of KwaZulu-Natal, South Africa}

\section{Introduction:}

- Data on the prevalence of low serum testosterone in men with type 2 diabetes mellitus (T2DM) from sub-Saharan Africa are scant

Aim:

- To determine the prevalence and associated risk factors of low serum testosterone in South African men with T2DM

- To determine the prevalence of androgen deficiency symptoms

Methods:

- Cross-sectional study in men with T2DM attending two diabetes clinics in Durban.

- Androgen deficiency symptoms (ADS) were assessed using Ageing Male's Symptom Scale (AMS) questionnaire and direct enquiry

- Serum total testosterone (TT), sex-hormone binding globulin (SHBG), luteinising hormone (LH), HbAlc, fructosamine and serum lipids were measured; free-testosterone (fT) was calculated with a standard formula.

- Control group included men without a history of diabetes

- Low serum fT (LSFT) was defined as $<180 \mathrm{pmol} / \mathrm{l}$ and low TT (LSTT) as < 12nmol/1/.

Results:

A. Characteristics

- The Study Group included 148 men with T2DM [African (Black) (58.7\%); Indians (39.2\%); Whites (2.1\%)] and Control Group included 50 men without a history of diabetes

\begin{tabular}{llll}
\hline & Study group & Control group & $\mathrm{p}$ \\
\hline Clinical characteristics & & & \\
Age (years) & $57.7 \pm 11.1$ & $43.9 \pm 10.7$ & $<0.001$ \\
BMI $\left(\mathrm{kg} / \mathrm{m}^{2}\right)$ & $29.82 \pm 6.0$ & $27.11 \pm 4.2$ & 0.011 \\
Diabetes duration (years) & $11.4 \pm 8.9$ & - & - \\
Laboratory results & & & \\
Total testosterone (nmol/l) & $14.5 \pm 5.8$ & $18.83 \pm 7.2$ & $<0.001$ \\
SHBG (nmol/l) & $40.7 \pm 20.3$ & $42.0 \pm 20.0$ & 0.685 \\
Free testosterone (pmol/l) & $265.9 \pm 90.4$ & $351.7 \pm 127.3$ & $<0.001$ \\
LH (IU/L) & $5.8 \pm 3.1$ & $4.5 \pm 2.3$ & 0.006 \\
\hline
\end{tabular}

\begin{tabular}{lll}
\hline & \multicolumn{2}{l}{ Study group $(\mathrm{n}=148)$} \\
\hline Symptoms on Enquiry & $\mathrm{n}$ & $\%$ \\
\hline Any symptom & 102 & 68.9 \\
Erectile dysfunction & 87 & 58.8 \\
Low libido & 83 & 56.1 \\
Decrease in beard growth & 4 & 2.7 \\
Infertility & 3 & 2.0 \\
\hline AMS questionnaire score $\geq 27$ & 111 & 75.0 \\
\hline
\end{tabular}
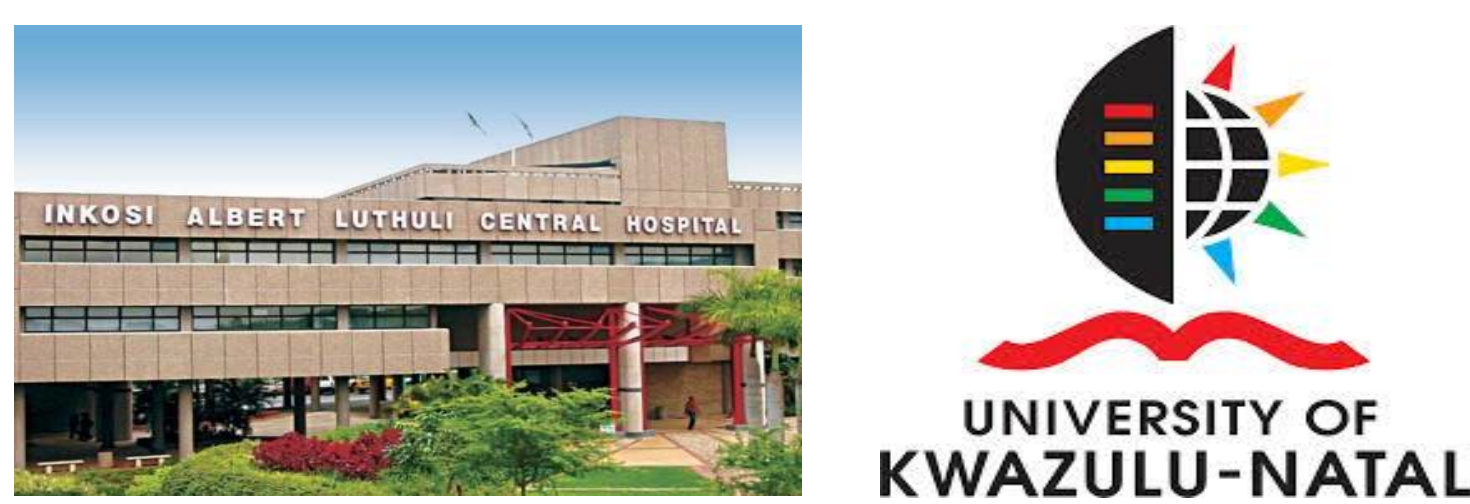

Acknowledgments and Funding

Approval to conduct this research was provided by the KZN Department of Health, Health Knowledge and Research Management and by the management at Inkosi Albert Luthuli Hospital. This study was made possible due to funding from Bayer (South Africa) and with assistance from Lancet Laboratories.
Results (continued)

B. Prevalence of low serum testosterone

\begin{tabular}{lcccc}
\hline & & Study Group(n:148) & Control Group $(\mathrm{n}: 50)$ & $\mathrm{p}^{*}$ \\
\hline LSTT & $\%(\mathrm{n})$ & $35.8(53)$ & $16.0(8)$ & 0.0087 \\
LSFT & $\%(\mathrm{n})$ & $16.2(24)$ & $2.0(1)$ & 0.0088 \\
\hline
\end{tabular}

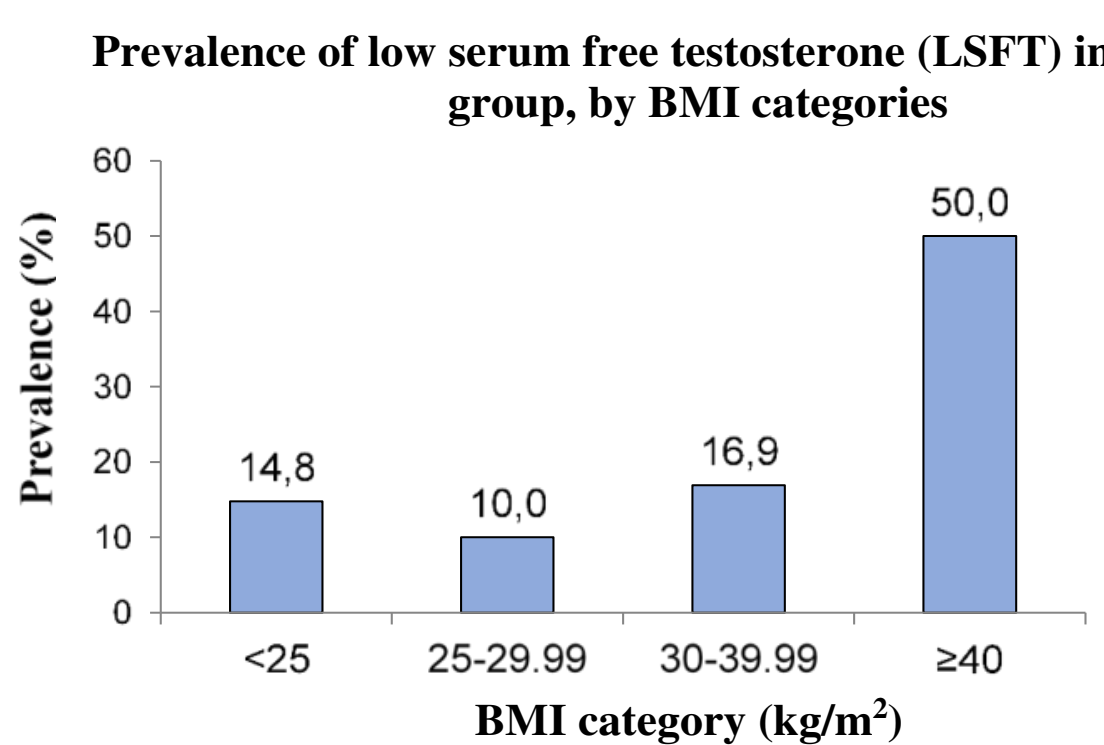

Serum free-testosterone in the study group, by age

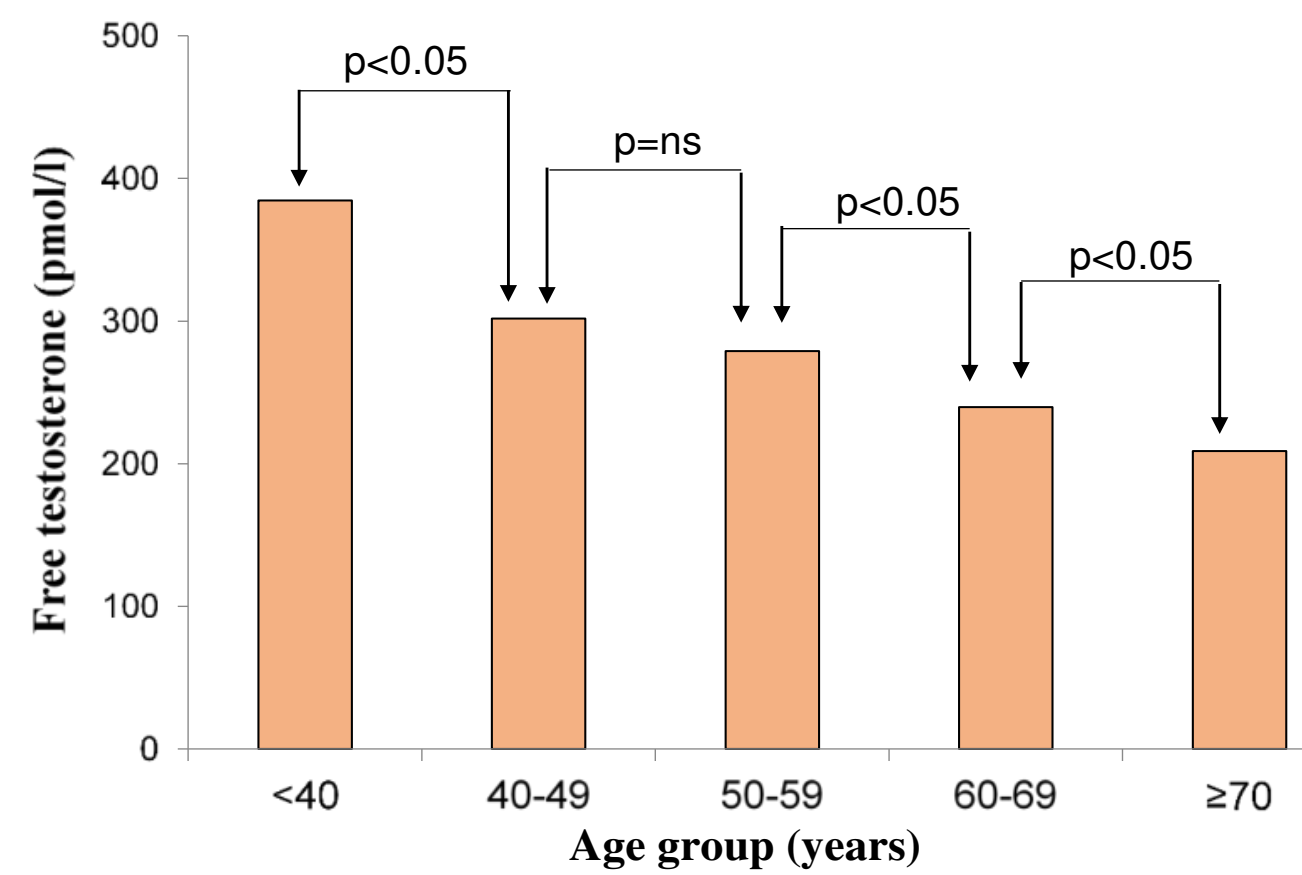

- In patients with LSFT ( $\mathrm{n}=24), 20.8 \%$ had elevated LH values, 70.8\% had normal levels and $8.3 \%$ had low LH levels.

C. Multivariate and Correlation Analysis

$>$ LSFT was significantly associated with age [OR 1.05 (95\%CI 1.02$1.218), \mathrm{p}=0.043)]$ and waist circumference [OR $1.033(95 \% \mathrm{CI} 0.999$ $1.068, \mathrm{p}=0.059)$ ] whilst LSTT was associated with BMI [OR 1.138 $(95 \%$ CI $1.063-1.218), \mathrm{p}<0.0001)]$

- Inverse correlation between TT and BMI $(\mathrm{r}-0.34, \mathrm{p}<0.001)$ and between TT and waist circumference $(\mathrm{r}-0.35, \mathrm{p}<0.001)$.

- fT correlated inversely with BMI, waist circumference and WHR ( $\mathrm{r}-0.27, \mathrm{p}<0.001 ; \mathrm{r}-0.33, \mathrm{p}<0.00 ; 1 \mathrm{r}-0.22, \mathrm{p}<0.05)$.

- Low correlation between AMS score and LSTT or LSFT.

Conclusion

- There is a high prevalence of low serum testosterone and ADS in this group of South African men with T2DM, similar to that reported in other countries.

- Serum Testosterone was lower in men with T2DM compared to controls

- There was a poor association and correlation between ADS and low testosterone.

- More data is required from South Africa and sub-Saharan Africa before routine screening can be recommended. 